\title{
An outbreak of leptospirosis among reserve military recruits, Hulu Perdik, Malaysia
}

\begin{abstract}
Here, we investigated an outbreak of leptospirosis among reserve military recruits that occurred following a survival exercise in the Hulu Perdik forest within the Hulu Langat district, Kuala Lumpur, Malaysia. Blood samples from the 12 patients that presented symptoms for febrile illness on clinical examination were subjected to laboratory investigation, comprising Lepto IgM rapid test, IgM ELISA, and microscopic agglutination test (MAT). All these patients were interviewed for possible risk factors for leptospirosis. Rodent trapping and environmental sampling for possible isolation of leptospires in the outbreak site was performed. The isolated leptospires were genetically characterized and investigated for the potential epidemiological link with human leptospirosis. Among the 12 patients, two $(2 / 12 ; 16.6 \%)$ were confirmed positive for leptospirosis by microscopic agglutination test (MAT with titers 400-800; serovar autumnalis and hardjobovis). Two Leptospira species from rodents (L. interrogans and L. borgpetersenii) and two from the environment (L. kmetyi and L. wolffii) were identified. The possible epidemiological link between human serovars and animal Leptospira species indicates rodents as the potential reservoir while the environment (soil and water) serves as a transmission route. This investigation highlights the robust presence of pathogenic leptospires on Malaysian environment and rodents which may present the risk of infection, especially among high-risk individuals. Hence, occupational risk individuals are cautioned to observe appropriate preventive measures including prophylaxis and seek immediate medical attention for any illness following similar activities.
\end{abstract}

Keyword: Emerging infectious disease; Leptospirosis; Malaysia; Occupational risk; Outbreak; Recreational exposure 